\title{
Microbiology of Meropenem-Vaborbactam: A Novel Carbapenem Beta-Lactamase Inhibitor Combination for Carbapenem-Resistant Enterobacterales Infections
}

Tanaya Bhowmick · Melvin P. Weinstein

Received: July 16, 2020 / Accepted: September 23, 2020 / Published online: October 5, 2020

(C) The Author(s) 2020

\section{ABSTRACT}

Vaborbactam is a novel boron-based beta-lactamase inhibitor developed to be effective against Klebsiella pneumoniae carbapenemase (KPC)-producing bacteria. This enzyme is a key driver in the global spread of $\beta$-lactam resistance among carbapenem-resistant Enterobacterales. Alone, vaborbactam has no antibacterial activity; however, the combination of meropenemvaborbactam has enhanced activity against gram-negative organisms, particularly Enterobacterales with class $\mathrm{A}$ and $\mathrm{C}$ carbapenemases. Multiple in vitro studies evaluating isolates from various geographic regions, and over different time periods, have demonstrated the high potency of meropenem-vaborbactam against organisms containing KPC2 and KPC3. However, meropenem-vaborbactam does not have activity against OXA-48 or metallo-beta lactamases. This review covers the in vitro studies of meropenem-vaborbactam performed to date, which evaluated both large cohorts of clinical isolates and engineered isolates, to

T. Bhowmick $(\varangle) \cdot$ M. P. Weinstein Department of Medicine, Rutgers Robert Wood Johnson Medical School, New Brunswick, NJ, USA e-mail: bhowmita@rwjms.rutgers.edu

\section{P. Weinstein}

Department of Pathology and Laboratory Medicine, Rutgers Robert Wood Johnson Medical School, New Brunswick, NJ, USA determine efficacy in various settings, including the presence of porin mutations and efflux pump upregulation.

\section{PLAIN LANGUAGE SUMMARY}

Meropenem-vaborbactam is a new combination antibiotic that was designed specifically for efficacy against bacteria that produce the Klebsiella pneumoniae carbapenemase (KPC) enzyme, which enables resistance to beta-lactam antibiotics. The global spread and increase of difficult-to-treat infections caused by carbapenem-resistant Enterobacterales (CRE) is in part because they produce KPC enzymes. The authors review the in vitro studies of meropenem-vaborbactam activity, which have included isolates from different geographic regions, time periods, and settings, showing that it has high potency against organisms containing KPC enzymes-KPC2 and KPC3. Meropenem-vaborbactam was tested against globally sourced isolates that carried different resistance mechanisms, including carbapenem resistance, multidrug resistant (MDR), and resistance to colistin and/or tigecycline; it inhibited activity of $99.1 \%$ Enterobacterales isolates tested at $\leq 1 \mu \mathrm{g} / \mathrm{ml}$, and at $\leq 8 \mu \mathrm{g} / \mathrm{ml}$ it inhibited $96.5 \%$ of MDR isolates and $82 \%$ of XDR isolates. Against OXA-48 or metallo-beta 
lactamase enzymes, meropenem-vaborbactam has limited or no activity, so in the Asia-Pacific region where MLBs are prevalent it was least effective, but and was most effective against US strains where KPC is prevalent. In multiple studies, meropenem-vaborbactam showed strong in vitro activity against E. coli, Enterobacter spp., and K. pneumoniae. Compared to available antibiotics, against both clinical and engineered isolates, as well as engineered E. coli strains with KPC, SHV, and TEM enzymes, meropenem-vaborbactam demonstrated lower MIC values. Overall, in vitro studies of meropenem-vaborbactam have shown enhanced activity against CRE and KPC producers compared to other antibiotics, which is needed in the current CRE environment where KPC is dominant.

Keywords: B-lactam; B-lactamase inhibitor; CRE; Meropenem-vaborbactam

\section{Key Summary Points}

Meropenem-vaborbactam as a combination has demonstrated enhanced in vitro activity against gram-negative organisms, particularly Enterobacterales with class A and C carbapenemases.

$\mathrm{KPC}$ is the most dominant strain of carbapenemase-resistant Enterobacterales (CRE). Meropenem-vaborbactam has been shown in multiple in vitro studies of globally sourced isolates to be a potent inhibitor of Enterobacterales with KPC enzymes.

Meropenem-vaborbactam does not have activity against OXA-48 or metallo-beta lactamases, thus was least effective in strains from the Asia-Pacific region where MLBs are prevalent and was most effective against strains from the US.
Compared to currently available antibiotics, meropenem-vaborbactam demonstrated lower MIC values against both clinical and engineered isolates, including engineered $E$. coli strains that had KPC, SHV, and TEM enzymes.

Sub-analysis in the TANGOII trial demonstrated meropenem-vaborbactam had a lower potential for resistance to develop.

\section{DIGITAL FEATURES}

This article is published with digital features, including a summary slide and plain language summary to facilitate understanding of the article. To view digital features for this article go to $\quad$ https://doi.org/10.6084/m9.figshare. 12937256.

\section{INTRODUCTION}

With the emergence of carbapenem-resistant Enterobacteriaceae (CRE) organisms, the need for new compounds with activity against these resistant isolates has become acute [1]. A successful method to overcome resistance mediated by beta-lactamase enzymes is to combine beta-lactams with $\beta$-lactamase inhibitors [2]; commonly used $\beta$-lactamase inhibitors are tazobactam, sulbactam, and clavulanic acid. The addition of these inhibitors to already established beta-lactams has produced the following combinations: piperacillin-tazobactam, ampicillin-sulbactam, and amoxicillin-clavulanate [3]. These combinations maintain the efficacy of the $\beta$-lactam class of antibiotics and expand the spectrum of activity against gramnegative pathogens $[4,5]$. However, these $\beta$ lactam inhibitors are only active against some Class A, SHV, TEM, Klebsiella pneumoniae carbapenemase (KPC) enzymes; none of these $\beta$ lactamase inhibitors have activity against the emerging and more resistant Class B (NDM IMP, 
VIM), C (not true carbapenemase), or D (OXAs) enzymes [6].

Avibactam is a diazabicyclooctane inhibitor, which is not based on the beta-lactam class and uses a urea core. Originally developed to target class A enzymes, Avibactam was found to have activity against enzymes in classes $\mathrm{A}, \mathrm{C}$, and a subset of D, specifically against KPC [7]. The combination of ceftazidime and avibactam is approved by the Federal Drug Administration (FDA) for the treatment of complicated intraabdominal infections, complicated urinary tract infections, hospital-acquired bacterial pneumonia, and ventilator-associated bacterial pneumonia. Unfortunately, development of resistant organisms after treatment with ceftazidime-avibactam has already been reported [8-11], fueling the need for and highlighting the importance of continued drug discovery and development.

Vaborbactam is a novel $\beta$-lactamase inhibitor based on a boron ring structure, giving it superior efficacy compared to older $\beta$-lactamase inhibitors and the recently approved avibactam [12]. Boronic acid has long been known as a safe compound with therapeutic potential for treatment of various diseases processes [13, 14]. Thus, it is not surprising that the development of a $\beta$-lactamase inhibitor with a boron core would be a potent agent. Boronic acids are unique in that the covalent structures that are formed with serine hydrolases can take the tetrahedral structure of either the acylation and deacylation states.

Vaborbactam contains a cyclic $\alpha$-acylaminoboronic acid, which forms a boronic ester ring that forces a preferred conformation for binding structure that results in greater potency [15]. Fortunately, it does not have any activity against mammalian serine proteases [15]. The compound was designed to form an aromatic ring, which would increase the affinity for $\beta$-lactamases as well as maintain the formed structure as the bond is reversible. Several candidate structures were docked with Classes A, C, and $\mathrm{D} \beta$-lactamases and evaluated; the structure that had favorable pre-covalent and covalent bonding for Classes A and C enzyme active sites was selected for further analysis. Subsequently, analogs of this structure were evaluated to determine the $\mathrm{N}$-acyl-substituent relationships to the potentiation of cephalosporins and carbapenems. The most potent analog was selected, and additional testing with crystallography confirmed visualization of a covalent bond between the serine residue of enzymes (amp C and CTX-m-15) with the inhibitor's boron atom. The selected analog was initially entitled RPX7009 and later named vaborbactam.

Of note, vaborbactam tested alone against Enterobacterales showed no activity with minimum inhibitory concentration (MIC) $)_{50}$ and $\mathrm{MIC}_{90}$ values of $>64 \mathrm{ug} / \mathrm{ml}$, indicating a lack of antibacterial activity [16].

The formulation of vaborbactam was tested with cephalosporins and aztreonam, but carbapenems produced the most potent combination, and meropenem with vaborbactam proved to be the most effective with the maximum potentiation [12, 17, 18]. Meropenem-vaborbactam was specifically designed to be effective against multidrug-resistant organisms, Enterobacterales-producing extended spectrum beta lactamases (ESBL), and carbapenemase-producing bacteria, such as (KPC) [16, 19-22]. This meropenem-vaborbactam combination maintains the broad spectrum of activity of meropenem, which includes many antibioticresistant gram-negative bacteria, and also utilizes the potent carbapenemase and $\beta$-lactamase inhibitor actions of vaborbactam, enhancing its range and potency of antimicrobial activity [16, 19]. This article is based on previously conducted studies and does not contain any studies with human participants or animals performed by any of the authors.

\section{MICROBIOLOGICAL PROPERTIES OF MEROPENEM-VABORBACTAM}

\section{Gram-Positive Organisms}

The combination meropenem-vaborbactam has not been evaluated in vitro against gram-positive organisms, but retains the gram-positive activity of meropenem; however, it has limited coverage against methicillin-resistant Staphylococcus aureus (MRSA), Enterococcus faecalis, and Enterococcus faecium [23-25]. 


\section{Gram-Negative Organisms}

Meropenem and vaborbactam individually have minimal and no activity, respectively, against KPC isolates [16]; however, the combination meropenem-vaborbactam is highly effective against gram-negative Enterobacterales, particularly those with KPC enzymes. This has been demonstrated by in vivo animal studies [15]. Moreover, several studies have evaluated meropenem-vaborbactam efficacy against clinical strains using reference broth microdilution techniques. Vaborbactam is a narrow-spectrum beta-lactamase inhibitor, which does not infer any additional protection against Class C/AmpC hyper production, which tends to cause resistance in Pseudomonas. However, one in vitro study suggested that vaborbactam may enhance activity against Pseudomonas aeruginosa, although the full findings have not yet been published [26]. Of note, meropenemvaborbactam has no improved activity against Acinetobacter baumannii and is not effective against the metallo-beta-lactamase (MBL) (Class B)-containing Enterobacterales [17, 27, 28].

Castanheira et al. evaluated the effectiveness of meropenem-vaborbactam against 315 clinical isolates of serine carbapenemase-producing Enterobacterales collected over a period of 13 years from multiple countries [16]. The long time interval and geographic variety were purposely chosen to diversify the test population. The majority of the isolates evaluated were $K$. pneumoniae (66\%), and the most common resistance genes detected among the Enterobacterales were bla $\mathrm{KPC}_{\mathrm{KP}}(46 \%)$ and $\mathrm{bla}_{\mathrm{KPC}-3}$ $(37 \%)$. When the isolates were tested alone against meropenem, only $2.2 \%$ of the entire collection were susceptible at the CLSI (Clinical \& Laboratory Standards Institute) breakpoint of $\leq 1$ for meropenem [29]. The combination of meropenem $(\leq 2 \mu \mathrm{g} / \mathrm{ml})$ with increasing concentrations of vaborbactam ranging from 4 to $32 \mu \mathrm{g} / \mathrm{ml}$ inhibited $90.2-98.1 \%$ of the isolates tested. Vaborbactam increased the activity of meropenem by at least 64 fold. When focusing on KPC-producing isolates, meropenem-vaborbactam with an inhibitor concentration of $8 \mu \mathrm{g} /$ $\mathrm{ml}$ inhibited 96.6\% K.pneumoniae and 100\% Escherichia coli, Enterobacter cloacae, Klebsiella oxytoca, Serratia marcescens, and Citrobacter freundii isolates. In addition, $98.7 \%$ of isolates that carried additional beta-lactamases were suppressed with the combination. Of the seven isolates that exhibited intrinsic resistance to meropenem-vaborbactam $\quad$ MICs $\geq 16 \mu \mathrm{g} / \mathrm{ml}$, four carried MBLs for VIM in addition to KPC, and the remaining three isolates showed alterations in expression of outer membrane porin (OMP) and efflux pump mechanisms (Table 1).

Optimal vaborbactam concentration was $8 \mu \mathrm{g} / \mathrm{ml}$, based on in vitro activity achieving adequate susceptibility at $97.8 \%$ of the tested isolates. Increasing dose did not achieve significantly higher susceptibility. More importantly, this concentration is roughly equivalent to the concentration of the FDA-approved dose of $2 \mathrm{~g}$ for vaborbactam (Table 2).

Subsequently, Castenheira et al. published a larger study that evaluated 14,304 worldwide contemporary gram-negative clinical isolates against meropenem-vaborbactam and comparator antibiotics [17]. The isolates were collected in 2014 from 82 hospitals as part of the SENTRY Antimicrobial Surveillance Program and included isolates that carried different resistance mechanisms so that some of the isolates were carbapenem resistant while others were multidrug resistant (MDR), including resistance to colistin and/or tigecycline. Meropenem effectively inhibited activity of $97.3 \%$ of Enterobacterales isolates tested at $\leq 1 \mu \mathrm{g} / \mathrm{ml}$. This value increased to $99.1 \%$ when the same isolates were tested against meropenem-vaborbactam at the same concentration and $99.6 \%$ for a concentration of $\leq 8 \mu \mathrm{g} / \mathrm{ml}$. There were 265 CRE isolates in this cohort, of which the majority $(79.6 \%)$ were $K$. pneumoniae. One hundred thirty-five of the 265 screened CRE isolates carried bla $\mathrm{KPC}_{\mathrm{KP}}$ genes. Meropenemvaborbactam had the most activity of the $\beta$ lactam agents tested against these CRE isolates and inhibited $84.2 \%$ of isolates at $\leq 8 \mu \mathrm{g} / \mathrm{ml}$, and against comparator agents high susceptibility rates were seen: amikacin $(56.2 \%)$ and colistin (70.3\%). Only tigecycline had higher susceptibility at $99.2 \%$ (Fig. 1).

The meropenem-vaborbactam $\mathrm{MIC}_{50} / \mathrm{MIC}_{90}$ for MDR isolates was $0.03 / 1 \mu \mathrm{g} / \mathrm{ml}$ and for 
Table 1 In vitro activity of meropenem-vaborbactam against clinical isolates from various cohorts

\begin{tabular}{|c|c|c|c|c|c|}
\hline & \multirow[t]{3}{*}{ No. of isolates tested } & \multicolumn{3}{|c|}{ Meropenem-vaborbactam $(8 \mu \mathrm{g} / \mathrm{ml})$} & \multirow[t]{3}{*}{ Ref } \\
\hline & & \multicolumn{3}{|l|}{$\mathrm{MIC}(\mu \mathrm{g} / \mathrm{ml})$} & \\
\hline & & Range & $50 \%$ & $90 \%$ & \\
\hline Enterobacterales & 10,426 & $\leq 0.015$ to $>32$ & $\leq 0.015$ & 0.06 & {$[17]$} \\
\hline Enterobacterales (KPC) & 991 & $\leq 0.03$ to $>32$ & 0.06 & 1 & {$[19]$} \\
\hline Escherichia coli & 4238 & $\leq 0.015$ to 32 & $\leq 0.015$ & $\leq 0.015$ & {$[17]$} \\
\hline Escherichia coli (KPC) & 35 & $\leq 0.03$ to $>32$ & $\leq 0.03$ & $\leq 0.03$ & {$[19]$} \\
\hline Escherichia coli (KPC) & 21 & $\leq 0.06$ & $\leq 0.06$ & $\leq 0.06$ & {$[16]$} \\
\hline Klebsiella pneumoniae & 2010 & $\leq 0.015$ to $>32$ & 0.03 & 0.12 & {$[17]$} \\
\hline Klebsiella pneumoniae (KPC) & 878 & $\leq 0.03$ to 0.12 & 0.12 & 1 & {$[19]$} \\
\hline Klebsiella pneumoniae (KPC) & 208 & $\leq 0.06$ to $>64$ & $\leq 0.06$ & 1 & {$[16]$} \\
\hline Klebsiella pneumoniae (KPC) & 121 & $\leq 0.004 / 8$ to $>64 / 8$ & .03 & .5 & {$[27]$} \\
\hline Klebsiella oxytoca & 429 & $\leq 0.015$ to 16 & 0.03 & 0.03 & {$[17]$} \\
\hline Klebsiella oxytoca (KPC) & 19 & $\leq 0.03$ to 0.12 & $\leq 0.03$ & 0.25 & {$[19]$} \\
\hline Klebsiella oxytoca (KPC) & 14 & $\leq 0.06$ to 2 & $\leq 0.06$ & 0.5 & {$[16]$} \\
\hline Enterobacter cloacae species complex & 950 & $\leq 0.015$ to 8 & $\leq 0.015$ & 0.03 & {$[17]$} \\
\hline E. cloacae (serine carpbapenemase) & 39 & $\leq 0.06$ to 4 & $\leq 0.06$ & 0.25 & {$[16]$} \\
\hline Enterobacter aerogenes & 355 & $\leq 0.015$ to 2 & 0.03 & 0.03 & {$[17]$} \\
\hline Enterobacter species (KPC) & 29 & $\leq 0.03$ to 0.12 & $\leq 0.03$ & 0.12 & {$[19]$} \\
\hline Citrobacter freundii species complex & 276 & $\leq 0.015$ to 8 & $\leq 0.015$ & 0.03 & {$[17]$} \\
\hline Citrobacter freundii (KPC) & 12 & $\leq 0.06$ to 0.25 & $\leq 0.06$ & 0.12 & {$[16]$} \\
\hline Citrobacter koseri & 194 & $\leq 0.015$ to 0.03 & $\leq 0.015$ & 0.03 & {$[17]$} \\
\hline Citrobacter species & 13 & $\leq 0.03$ to 0.12 & $\leq 0.03$ & 0.06 & {$[19]$} \\
\hline Proteus mirabilis & 525 & $\leq 0.015$ to 1 & 0.06 & 0.12 & {$[17]$} \\
\hline Indole-positive Proteeae spp. & 585 & $\leq 0.015$ to $>32$ & 0.06 & 0.06 & {$[17]$} \\
\hline Serratia marcescens & 666 & $\leq 0.015$ to 32 & 0.03 & 0.06 & {$[17]$} \\
\hline Serratia marcescens (KPC) & 16 & $\leq 0.03$ to 2 & 0.06 & 1 & {$[19]$} \\
\hline CRE & 265 & $\leq 0.015$ to $>32$ & 0.5 & 32 & {$[17]$} \\
\hline CRE & 330 & $\leq 0.015$ to $>32$ & 0.5 & 32 & {$[30]$} \\
\hline KPC producers & 135 & $\leq 0.015$ to 8 & 12 & 0.5 & {$[17]$} \\
\hline KPC producers & 206 & $\leq 0.015$ to 32 & 0.25 & 1 & {$[27,30]$} \\
\hline Non-KPC-producing CRE & 129 & $\leq 0.015$ to $>32$ & 4 & $>32$ & {$[17]$} \\
\hline Non-KPC-producing CRE & 121 & 0.25 to $>32$ & 16 & $>32$ & \\
\hline Carbapenemase-negative isolates & 63 & $\leq 0.015$ to 32 & 1 & 4 & {$[17]$} \\
\hline
\end{tabular}


Table 1 continued

\begin{tabular}{llllll}
\hline & No. of isolates tested & \multicolumn{2}{l}{ Meropenem-vaborbactam $(\mathbf{8} \boldsymbol{\mu g} / \mathbf{m l})$} & \multirow{2}{*}{ Ref } \\
\cline { 3 - 4 } & & \multicolumn{2}{l}{ MIC $(\boldsymbol{\mu g} / \mathbf{m l})$} & & \\
\cline { 3 - 4 } & & Range & $\mathbf{5 0 \%}$ & $\mathbf{9 0 \%}$ & \\
\hline Carbapenemase-negative isolates & 38 & 0.25 to 32 & 2 & 16 & {$[30]$} \\
MDR & 1210 & $\leq 0.015$ to $>32$ & 0.03 & 1 & {$[17]$} \\
XDR & 161 & $\leq 0.015$ to $>32$ & 0.5 & 32 & {$[17]$} \\
MBL producer & 41 & 1 to $>32$ & 32 & $>32$ & {$[17]$} \\
MBL producer & 52 & 1 to $>32$ & 32 & $>32$ & {$[30]$} \\
Pseudomonas aeruginosa & 2604 & $\leq 0.015$ to $>32$ & 0.5 & 8 & {$[17]$} \\
Pseudomonas aeruginosa & 98 & $0.25 / 8$ to $64 / 8$ & 8 & 32 & {$[27]$} \\
\hline
\end{tabular}

Adapted from [16, 17, 19, 27, 30]

KPC Klebsiella pneumoniae carbapenemase, CRE carbapenem-resistant Enterobacterales, MDR multidrug resistant, XDR extensively drug resistant, $M B L$ metallo-beta lactamase

Table 2 Establish breakpoints for meropenem-vaborbactam against enterobacterales

\begin{tabular}{|c|c|c|c|c|c|c|}
\hline & \multicolumn{3}{|c|}{ Minimum inhibitory concentrations $(\mu \mathrm{g} / \mathrm{ml})$} & \multicolumn{3}{|c|}{ Disk diffusion (zone diameters in $\mathbf{m m}$ ) } \\
\hline & $S$ & $I$ & $R$ & $S$ & $I$ & $R$ \\
\hline FDA [36] & $\leq 4 / 8$ & $8 / 8$ & $\geq 16 / 8$ & $\geq 17$ & $14-16$ & $\leq 13$ \\
\hline CLSI [29] & $\leq 4 / 8$ & $8 / 8$ & $\geq 16 / 8$ & $\geq 18$ & $15-17$ & $\leq 14$ \\
\hline EUCAST [37] & $\leq 8 / 8$ & - & $>8 / 8$ & * & * & * \\
\hline
\end{tabular}

${ }^{*}$ EUCAST disk diffusion in preparation

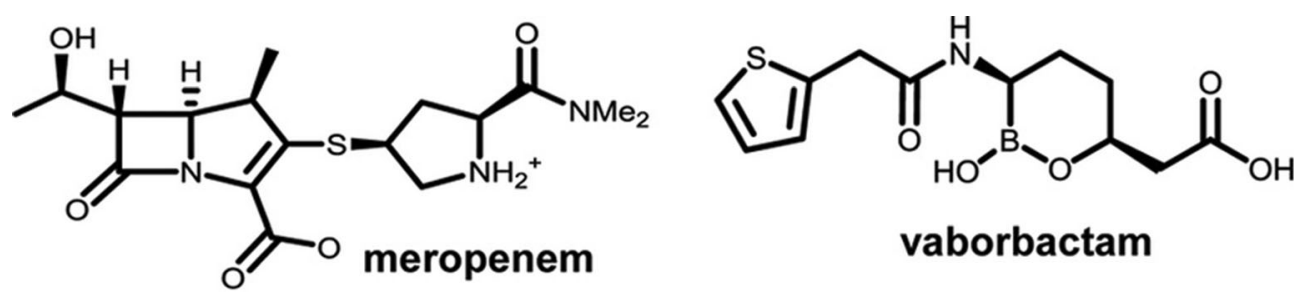

Fig. 1 Chemical structures of meropenem and vaborbactam. Me 2 dimethyl [12]. Reused with permission under the 'Creative Commons CC BY' license from [12]. Copyright (c) 2017, American Society for Microbiology

extensively drug-resistant (XDR) isolates was $0.5 / 32 \mu \mathrm{g} / \mathrm{ml}$. Meropenem-vaborbactam at $\leq 8 \mu \mathrm{g} / \mathrm{ml}$ inhibited $96.5 \%$ of $\mathrm{MDR}$ isolates and $82 \%$ of XDR isolates. Similar to the previous analysis, the study confirmed that meropenemvaborbactam has limited activity against MBLs. 
Among the $15.5 \%$ of the CRE isolates that contained MBLs, approximately two-thirds had the NDM-1 gene. OXA-48 also accounted for $10.2 \%$ of the CRE isolates. Meropenem-vaborbactam was most effective in strains from the US and least effective in strains from Asia-Pacific countries, likely due to the region's higher prevalence of MBLs. There were only seven isolates carrying carbapenemases from the Asia-Pacific region.

The same investigators utilized a 2015 worldwide collection of 11,559 Enterobacterales isolates to evaluate meropenem-vaborbactam activity, finding that meropenem-vaborbactam demonstrated enhanced activity against CRE and KPC producers compared to other antibiotics [30]. Meropenem alone inhibited $96.9 \%$ of all the Enterobacterales tested, which increased to $99.3 \%$ when the isolates were tested against the combination of meropenem-vaborbactam. Overall, $97 \%$ of $K$. pneumoniae isolates were inhibited by meropenem-vaborbactam. Of the 330 CRE isolates in the cohort, the majority $(80.6 \%)$ were K. pneumoniae, $88.5 \%$ had carbapenemase genes, $15.7 \%$ had MBL genes, and $13.3 \%$ had OXA-48 genes. Meropenem-vaborbactam at $\leq 4 / 8 \mu \mathrm{g} / \mathrm{ml}$ inhibited $73.9 \%$ of the CRE Enterobacterales and only $31.4 \%$ of the non-KPC gene CRE isolates. The one KPC isolate $(0.5 \%)$ that was not inhibited by meropenemvaborbactam contained a porin mutation.

Lapuebla et al. evaluated 4500 gram-negative clinical isolates from 11 New York City hospitals against meropenem-vaborbactam, meropenem, and other antibiotics [27]. Meropenem-vaborbactam had strong in vitro activity against E. coli, Enterobacter spp., and K. pneumoniae and enhanced activity in KPC-producing CR isolates. Among E. coli isolates $b l a_{\mathrm{KPC}}$ was the only carbapenemase gene detected, and all isolates were susceptible to meropenem-vaborbactam at $\leq 8 \mu \mathrm{g} / \mathrm{ml}$. Eighty-eight percent of $K$. pneumoniae isolates were susceptible to meropenem, and $99.8 \%$ were susceptible to meropenemvaborbactam (with a vaborbactam concentration of $8 \mu \mathrm{g} / \mathrm{ml}$ ). Meropenem-vaborbactam had activity against $119 / 121(98.3 \%)$ of the KPC $K$. pneumoniae strains. In addition, $26 \mathrm{~K}$. pneumoniae isolates with known resistance mechanisms expressing bla $_{\mathrm{KPC}}, a c r \mathrm{~B}$, and ompK36 were tested; only 2 (both with decreased expression of ompK36) had elevated MIC $>64 \mu \mathrm{g} / \mathrm{ml}$ to meropenem, which decreased to 1 and 2 with the addition of vaborbactam. All 211 isolates of Enterobacter species, 7 of which had the bla $\mathrm{KPC}_{\mathrm{K}}$ gene, were sensitive to the combination of meropenem-vaborbactam. Evaluation of $A$. baumannii and $P$. aeruginosa revealed no significant change among the non-susceptible meropenem isolates when tested against meropenem-vaborbactam, which was expected.

\section{Anaerobic Organisms}

Combination meropenem-vaborbactam retains the anaerobic activity of meropenem [31].

\section{MECHANISMS OF RESISTANCE AND LOWER POTENTIAL FOR DEVELOPMENT OF RESISTANCE}

Carbapenem-resistant Enterobacterales often have multiple mechanisms of resistance, such as porin-mutations or efflux pumps, in addition to carbapenemase-producing enzymes. To evaluate its potency in various situations, meropenem-vaborbactam was tested against engineered strains of $\beta$-lactamase-producing E. coli and K. pneumoniae with and without additional mutations [12]. The study demonstrated that an E. coli strain without any betalactamase inhibitor had an MIC of $\leq 0.03 \mu \mathrm{g} / \mathrm{ml}$ for both meropenem and meropenem-vaborbactam, which was $\leq 0.125 \mu \mathrm{g} / \mathrm{ml}$ for both aztreonam \pm beta-lactamase inhibitor and ceftazidime \pm beta-lactamase inhibitor (beta-lactamase inhibitors tested were vaborbactam, tazobactam, and clavulanic acid). The trend for lower MIC values with meropenem-vaborbactam was also observed with engineered $E$. coli strains that had KPC, SHV, and TEM enzymes. Moreover, the addition of vaborbactam to ceftazidime and aztreonam restored the activity against strains that contained class A carbapenemases with lower MIC values compared to the other $\beta$-lactamase inhibitors. For extended-spectrum $\beta$-lactamase (ESBL) genes TEM 
and SHV, the MIC value was lower with the addition of vaborbactam to the tested beta-lactam compared to the $\beta$-lactam alone; however, tazobactam and clavulanic acid proved to be more potent than vaborbactam. In the engineered $K$. pneumoniae isolates, the investigators produced isolates that had both a porin mutation and a $\beta$-lactamase, resulting in increased MIC values for meropenem (compared to wildtype strains), which decreased with the addition of vaborbactam for KPC $(64 \mu \mathrm{g} / \mathrm{ml} \rightarrow 2 \mu \mathrm{g} / \mathrm{ml})$, SHV $(0.5 \mu \mathrm{g} / \mathrm{ml} \rightarrow 0.25 \mu \mathrm{g} / \mathrm{ml})$, and ampC $(4 \mu \mathrm{g} /$ $\mathrm{ml} \rightarrow 1 \mu \mathrm{g} / \mathrm{ml})$, but not for OXA-48 $(64 \mu \mathrm{g} / \mathrm{ml} \rightarrow$ $64 \mu \mathrm{g} / \mathrm{ml}$ ), which was expected. Further evaluation of the effects of mutations of porin proteins (Ompk35 and Ompk36) and efflux pumps (AcrAB) revealed that efflux pumps alone had minimal effect, but the combination of one porin and efflux pump mutations resulted in a two- to four-fold increase of MIC, and the combination of both porin mutations resulted in 16-fold increased MIC. The study again demonstrated the lack of potency of meropenem-vaborbactam against NDM and VIM strains.

Another recently published study reported two newly described loss-of-function mutations for porin production. KvrA loss reduced OmpK35 and OmpK36 porin production, again demonstrating the importance of porin mutations on the effect of reducing susceptibility to meropenem-vaborbactam in a KPC-3-producing K. pneumoniae isolate [32].

Another in vitro study utilized CRE genotypes and laboratory-engineered $E$. coli isolates harboring mutant $b l a_{\mathrm{KPC}}$ genes, which are associated with ceftazidime-avibactam resistance, to identify meropenem-vaborbactam susceptibility and MICs [33].

Ninety-eight percent $(117 / 120)$ of CRE isolates were susceptible to meropenem-vaborbactam (MICs $\leq 4 \mu \mathrm{g} / \mathrm{ml}$ ), and median MICs were lower for meropenem-vaborbactam compared to ceftazidime-avibactam $(0.034 \mu \mathrm{g} / \mathrm{ml}$ and 1$)$. All bla $a_{\mathrm{KPC}}$-harboring K. pneumoniae isolates were susceptible to meropenem-vaborbactam, and the addition of vaborbactam reduced the MICs in $78 \%$ of isolates $(14 / 18)$. Both wild-type and variant KPC enzymes were inhibited by meropenem-vaborbactam. However, in KPC- producing $K$. pneumoniae isolates, meropenemvaborbactam MICs were higher in those isolates that displayed mutant ompK36 genes $(n=26)$ compared to wild-type ompK36 genes $(n=54)$ (0.25 versus $0.03 \mu \mathrm{g} / \mathrm{ml} ; P<0.0001)$.

The addition of vaborbactam at $8 \mu \mathrm{g} / \mathrm{ml}$ against E.Coli isolates containing wildtype $b l a_{\mathrm{KPC}}$ or mutant $b l a_{\mathrm{KPC}}$ lowered the meropenem MICs 2- to 512-fold, which resulted in meropenem-vaborbactam MICs of $0.03 \mu \mathrm{g} / \mathrm{ml}$. Thus, meropenem-vaborbactam has demonstrated in vitro activity against CRE, including isolates resistant to ceftazidime-avibactam.

The in vitro activity of meropenem-vaborbactam against 991 KPC-positive Enterobacterales isolates, collected globally from 2014 to 2015 [19], was compared to the activity of seven other antibiotic agents. Although the overall susceptibility of the meropenem-vaborbactam was similar to ceftazidime-avibactam ( $99 \%$ vs. 98.2\%), meropenem-vaborbactam achieved potency at lower $\mathrm{MIC}_{90}$ values of $1 \mu \mathrm{g} / \mathrm{ml} \mathrm{com-}$ pared to ceftazidime-avibactam at $4 \mu \mathrm{g} / \mathrm{ml}$. Similar results were seen between meropenemvaborbactam and ceftazidime-avibactam when organisms were evaluated at the species level using $\mathrm{MIC}_{90}$ values $(\mu \mathrm{g} / \mathrm{ml}) ; K$. pneumoniae $(1 \mu \mathrm{g} / \mathrm{ml}$ vs. $4 \mu \mathrm{g} / \mathrm{ml})$, E. coli $(\leq 0.03 \mu \mathrm{g} / \mathrm{ml}$ vs. $1.0 \mu \mathrm{g} / \mathrm{ml})$, Enterobacter $(0.12 \mu \mathrm{g} / \mathrm{ml}$ vs. $2 \mu \mathrm{g} / \mathrm{ml})$, K. oxytoca $(0.25 \mu \mathrm{g} / \mathrm{ml}$ vs. $4 \mu \mathrm{g} / \mathrm{ml}), S$. marcescens $(1 \mu \mathrm{g} / \mathrm{ml}$ vs. $2 \mu \mathrm{g} / \mathrm{ml})$ and Citrobacter spp $(0.06 \mu \mathrm{g} / \mathrm{ml}$ vs. $2 \mu \mathrm{g} / \mathrm{ml})$, respectively. Interestingly, further evaluation of isolates resistant to meropenem-vaborbactam and ceftazidime-avibactam revealed only $20.8 \%$ cross-resistance. Meropenem-vaborbactam was also superior to tigecycline, gentamicin, and polymixin $\mathrm{B}$.

Importantly, the lower potential for resistance to meropenem-vaborbactam to develop has been demonstrated clinically. In a subanalysis of the Targeting Antibiotic Non-susceptible Gram-negative Organisms (TANGO) II trial, 1 patient of 25 (4\%) with CRE K. pneumoniae treated with meropenem-vaborbactam for 6 days failed therapy with an increase in MIC from $0.25 \mu \mathrm{g} / \mathrm{ml}$ to $1.0 \mu \mathrm{g} / \mathrm{ml}$, retaining activity against meropenem-vaborbactam. Of the four patients treated with ceftazidime-avibactam monotherapy, one $(25 \%)$ had an increase in the MIC value of $K$. pneumoniae from $0.5 \mu \mathrm{g} / \mathrm{ml}$ 
to $>128 \mu \mathrm{g} / \mathrm{ml}$, resulting in non-susceptibility [34]. In a different retrospective study of patients treated for CRE infections, post-hoc analysis revealed that 3 of 105 patients treated with ceftazidime-avibactam vs. no patients of the 26 treated with meropenem-vaborbactam had a recurrent infection due to development of a resistance organism [35]. Although these evaluations are of small cohort of patients, it is an important observation demonstrating the lower potential for developing resistance.

In an effort to explore optimal combinations of antimicrobial agents with activity against serine and MBL-producing CROs, the activity of aztreonam plus ceftazidime-avibactam and aztreonam plus meropenem-vaborbactam against clinical E. coli and K. pneumoniae strains coproducing NDM and one or more serine $\beta$ lactamases was evaluated and compared in a recent in vitro study [6]. The addition of aztreonam with each of these combination agents resulted in synergistic activity against enterobacteriaceae strains that co-produce NDM and at least one serine $\beta$-lactamase, with the anticipated exception of aztreonam plus meropenemvaborbactam having no activity against OXA48-like-producing enterobacteriaceae strains. Future studies are needed to confirm and expand upon these findings, but these findings suggest that these combinations could be a possible treatment in patients with aztreonamresistant NDM and serine- $\beta$-lactamase-producing enterobacteriaceae infections.

\section{CONCLUSION}

In conclusion, meropenem-vaborbactam is a potent inhibitor of Enterobacterales with KPC enzymes. It exhibits lower MIC values with in vitro testing against both clinical and engineered isolates compared to currently available antibiotics. Although the combination does not provide any additional coverage for Pseudomonas, Acinetobacter, or Enterobacterales that have MBL enzymes, it is a welcome and needed agent in the current CRE environment, which consists of predominantly KPC-containing strains.

\section{ACKNOWLEDGEMENTS}

Funding. No funding was provided for the research or writing of this manuscript. The Rapid Service Fees were fully funded by Melinta Therapeutics.

Authorship. All named authors meet the International Committee of Medical Journal Editors (ICMJE) criteria for authorship for this article, take responsibility for the integrity of the work as a whole, and have given their approval for this version to be published.

Disclosures. Tanaya Bhowmick and Melvin P. Weinstein have no conflicts to disclose.

Compliance with Ethics Guidelines. This article is based on previously conducted studies and does not contain any studies with human participants or animals performed by any of the authors.

Open Access. This article is licensed under a Creative Commons Attribution-NonCommercial 4.0 International License, which permits any non-commercial use, sharing, adaptation, distribution and reproduction in any medium or format, as long as you give appropriate credit to the original author(s) and the source, provide a link to the Creative Commons licence, and indicate if changes were made. The images or other third party material in this article are included in the article's Creative Commons licence, unless indicated otherwise in a credit line to the material. If material is not included in the article's Creative Commons licence and your intended use is not permitted by statutory regulation or exceeds the permitted use, you will need to obtain permission directly from the copyright holder. To view a copy of this licence, visit http://creativecommons.org/licenses/by$\mathrm{nc} / 4.0 /$. 


\section{REFERENCES}

1. Lai CC, Chen CC, Tang HJ. Meropenem-vaborbactam in the treatment of acute bacterial infections. J Clin Med. 2019;8(10):1650.

2. Drawz SM, Papp-Wallace KM, Bonomo RA. New beta-lactamase inhibitors: a therapeutic renaissance in an MDR world. Antimicrob Agents Chemother. 2014;58(4):1835-46.

3. Letourneau A. Combination beta-lactamase inhibitors, carbapenems, and monobactams. In: Hooper D, Bloom A (Ed.) UpToDate, Waltham, MA. 2020.

4. Adam D. Beta-lactam/beta-lactamase inhibitor combinations in empiric management of pediatric infections. J Int Med Res. 2002;30(Suppl 1): 10A-A1919.

5. Finegold S. In vitro efficacy of betalactam/beta-lactamase inhibitor combinations against bacteria involved in mixed infections. Int $\mathrm{J}$ Antimicrob Agents. 1999;12:S9-S14.

6. Biagi M, Wu T, Lee M, Patel S, Butler D, Wenzler E. Exploring aztreonam in combination with ceftazidime-avibactam or meropenem-vaborbactam as potential treatments for metallo- and serine- $\beta$-lactamase-producing Enterobacteriaceae. Antimicrob Agents Chemother. 2019;63(12):e01426-e1519.

7. Shields RK, Clancy CJ, Hao B, et al. Effects of Klebsiella pneumoniae carbapenemase subtypes, extended-spectrum beta-lactamases, and porin mutations on the in vitro activity of ceftazidimeavibactam against carbapenem-resistant K. pneumoniae. Antimicrob Agents Chemother. 2015;59(9):5793-7.

8. Shields RK, Potoski BA, Haidar G, et al. Clinical outcomes, drug toxicity, and emergence of ceftazidime-avibactam resistance among patients treated for carbapenem-resistant enterobacteriaceae Infections. Clin Infect Dis. 2016;63(12):1615-8.

9. Shields RK, Nguyen MH, Press EG, Chen L, Kreiswirth BN, Clancy CJ. Emergence of ceftazidime-avibactam resistance and restoration of carbapenem susceptibility in klebsiella pneumoniae carbapenemase-producing $\mathrm{K}$ pneumoniae: a case report and review of literature. Open Forum Infect Dis. 2017;4(3): of 101.

10. Shields RK, Nguyen MH, Chen L, Press EG, Kreiswirth BN, Clancy CJ. Pneumonia and renal replacement therapy are risk factors for ceftazidime-avibactam treatment failures and resistance among patients with carbapenem-resistant Enterobacteriaceae infections. Antimicrob Agents Chemother. 2018;62(5):e02497-17.
11. Kawai A, McElheny CL, Iovleva A, et al. Structural basis of reduced susceptibility to ceftazidime-avibactam and cefiderocol in enterobacter cloacae due to AmpC R2 loop deletion. Antimicrob Agents Chemother. 2020:e00198-20.

12. Lomovskaya O, Sun D, Rubio-Aparicio D, et al. Vaborbactam: spectrum of beta-lactamase inhibition and impact of resistance mechanisms on activity in Enterobacteriaceae. Antimicrob Agents Chemother. 2017;61(11):e01443-e1517.

13. Baker SJ, Ding CZ, Akama T, Zhang YK, Hernandez $\mathrm{V}$, Xia Y. Therapeutic potential of boron-containing compounds. Future Med Chem. 2009;1(7):1275-88.

14. Beesley T, Gascoyne N, Knott-Hunziker V, et al. The inhibition of class $\mathrm{C}$ beta-lactamases by boronic acids. Biochem J. 1983;209(1):229-33.

15. Hecker SJ, Reddy KR, Totrov M, et al. Discovery of a cyclic boronic acid $\beta$-lactamase inhibitor (RPX7009) with utility vs Class A serine carbapenemases. J Med Chem. 2015;58(9):3682-92.

16. Castanheira M, Rhomberg PR, Flamm RK, Jones RN Effect of the $\beta$-lactamase inhibitor vaborbactam combined with meropenem against serine carbapenemase-producing Enterobacteriaceae. Antimicrob Agents Chemother. 2016;60(9):5454-8.

17. Castanheira M, Huband MD, Mendes RE, Flamm RK. Meropenem-vaborbactam tested against contemporary gram-negative isolates collected worldwide during 2014, including carbapenem-resistant, KPC-producing, multidrug-resistant, and extensively drug-resistant enterobacteriaceae. Antimicrob Agents Chemother. 2017;61(9):e00567-e617.

18. Livermore DM, Mushtaq S. Activity of biapenem (RPX2003) combined with the boronate $\beta$-lactamase inhibitor RPX7009 against carbapenem-resistant Enterobacteriaceae. J Antimicrob Chemother. 2013;68(8):1825-31.

19. Hackel MA, Lomovskaya O, Dudley MN, Karlowsky JA, Sahm DF. In vitro activity of meropenemvaborbactam against clinical isolates of KPC-positive Enterobacteriaceae. Antimicrob Agents Chemother. 2018;62(1):e01904-e1917.

20. Kinn PM, Chen DJ, Gihring TM, et al. In vitro evaluation of meropenem-vaborbactam against clinical CRE isolates at a tertiary care center with low KPC-mediated carbapenem resistance. Diagn Microbiol Infect Dis. 2019;93(3):258-60.

21. Sabet M, Tarazi Z, Griffith DC. Activity of meropenem-vaborbactam against pseudomonas aeruginosa and acinetobacter baumannii in a neutropenic mouse thigh infection model. 
Antimicrob Agents Chemother. 2018;63(1): e01665-e1718.

22. Savov E, Trifonova A, Kovachka K, Kjosseva E, Strateva T. Antimicrobial in vitro activities of ceftazidime-avibactam, meropenem-vaborbactam and plazomicin against multidrug-resistant Acinetobacter baumannii and Pseudomonas aeruginosa-a pilot Bulgarian study. Infect Dis (Lond). 2019;51(11-12): 870-3.

23. Baldwin CM, Lyseng-Williamson KA, Keam SJ. Meropenem. Drugs. 2008;68(6):803-38.

24. Lutsar I, Chazallon C, Trafojer U, et al. Meropenem vs standard of care for treatment of neonatal late onset sepsis (NeoMero1): a randomised controlled trial. PLoS ONE. 2020;15(3):e0229380.

25. Zhanel GG, Wiebe R, Dilay L, et al. Comparative review of the carbapenems. Drugs. 2007;67(7): 1027-52.

26. Patel TS, Kaye KS, Krishnan J, et al. 521. Comparative in vitro activity of meropenem/vaborbactam and meropenem against a collection of real-world clinical isolates of Pseudomonas aeruginosa. Open Forum Infect Di. 2019;6(Supplement_2):S251-S.

27. Lapuebla A, Abdallah M, Olafisoye O, et al. Activity of meropenem combined with RPX7009, a novel beta-lactamase inhibitor, against gram-negative clinical isolates in New York City. Antimicrob Agents Chemother. 2015;59(8):4856-60.

28. Castanheira M, Doyle TB, Kantro V, Mendes RE, Shortridge D. Meropenem-vaborbactam activity against carbapenem-resistant enterobacterales isolates collected in U.S. Hospitals during 2016 to 2018. Antimicrob Agents Chemother. 2020;64(2): e01951-19.

29. CLSI. Performance Standards for Antimicrobial Susceptibility Testing. 30th ed. CLSI Supplement M100. Wayne, PA: Clinical and Laboratory Standards Institute. 2020.

30. Pfaller MA, Huband MD, Mendes RE, Flamm RK, Castanheira M. In vitro activity of meropenem/ vaborbactam and characterisation of carbapenem resistance mechanisms among carbapenem-resistant Enterobacteriaceae from the 2015 meropenem/ vaborbactam surveillance programme. Int $\mathrm{J}$ Antimicrob Agents. 2018;52(2):144-50.

31. Goldstein EJC, Citron DM, Tyrrell KL, Merriam CV. In vitro activity of biapenem plus RPX7009, a carbapenem combined with a serine $\beta$-lactamase inhibitor, against anaerobic bacteria. Antimicrob Agents Chemother. 2013;57(6):2620-30.

32. Dulyayangkul P, Wan Nur Ismah WAK, Douglas EJA, Avison MB. Mutation of KVR causes OmpK35 and OmpK36 Porin Downregulation and reduced meropenem-vaborbactam susceptibility in KPCproducing Klebsiella pneumoniae. Antimicrob Agents Chemother. 2020;64(7):e02208-19.

33. Wilson WR, Kline EG, Jones CE, et al. Effects of KPC variant and Porin genotype on the in vitro activity of meropenem-vaborbactam against carbapenemresistant Enterobacteriaceae. Antimicrob Agents Chemother. 2019;63(3):e02048-e2118.

34. Lomovskaya O, Castanheira M, Vazquez J, et al. Assessment of MIC increases with meropenemvaborbactam and ceftazidime-avibactam in TANGO II (a Phase 3 Study of the Treatment of CRE Infections). Abstract No.1874. Open Forum Infect Dis. 2017;4(suppl_1):S540-S.

35. Ackley R, Roshdy D, Meredith J, et al. Meropenemvaborbactam versus ceftazidime-avibactam for treatment of carbapenem-resistant enterobacteriaceae infections. Antimicrob Agents Chemother. 2020;64(5):e02313-e2319.

36. The Medicines Company. VABOMERE ${ }^{\mathrm{TM}}$ (meropenem and vaborbactam) for injection, for intravenous use (Package Insert). 2019. https://www. accessdata.fda.gov/drugsatfda_docs/label/2017/ 209776lbl.pdf. Accessed 22 Aug 2019.

37. The European Committee on Antimicrobial Susceptibility Testing. Breakpoint tables for interpretation of MICs and zone diameters, version 10.0 2020. 